\title{
ON THE VALUES AT NEGATIVE HALF-INTEGERS OF THE DEDEKIND ZETA FUNCTION OF A REAL QUADRATIC FIELD
}

\author{
MINKING EIE
}

(Communicated by Larry J. Goldstein)

\begin{abstract}
The zeta function $\zeta(A, s)$ associated with a narrow ideal class $A$ for a real quadratic field can be decomposed into $\sum_{Q} Z_{Q}(s)$, where $Z_{Q}(s)$ is a Dirichlet series associated with a quadratic form $Q(x, y)=a x^{2}+b x y+c y^{2}$, and the summation is over finite reduced quadratic forms associated to the narrow ideal class $A$. The values of $Z_{Q}(s)$ at nonpositive integers were obtained by Zagier [16] and Shintani [12] via different methods. In this paper, we shall obtain the values of $Z_{Q}(s)$ at negative half-integers $s=-1 / 2,-3 / 2, \ldots,-m+$ $1 / 2, \ldots$. The values of $Z_{Q}(s)$ at nonpositive integers were also obtained by our method, and our results are consistent with those given in [16].
\end{abstract}

\section{INTRODUCTION}

Let $Q(x, y)=a x^{2}+b x y+c y^{2}$ be a binary quadratic form with integral coefficients and of discriminant $D=b^{2}-4 a c$. Also let $T=\left[\begin{array}{ll}\alpha \beta \\ \gamma & \delta\end{array}\right]$ be an element of $\mathrm{GL}_{2}(Z)$ with det $T=\alpha \delta-\beta \gamma= \pm 1$. Then $T$ acts on the collection of forms of discriminant $D$ by the action:

$$
Q \rightarrow Q \mid T(x, y)=(\alpha \delta-\beta \gamma) Q(\alpha x+\beta y, \gamma x+\delta y) .
$$

Two forms $Q_{1}$ and $Q_{2}$ are said to be equivalent in the narrow sense (resp. wide sense) if $Q_{1}=Q_{2} \mid T$ for some $T \in \mathrm{SL}_{2}(Z)$ (resp. $T \in \mathrm{GL}_{2}(Z)$ and $\operatorname{det} T= \pm 1)$. A quadratic form $Q(x, y)=a x^{2}+b x y+c y^{2}$ is called reduced (in the narrow sense) if $a>0, c>0$, and $b>a+c . Q$ is primitive if the g.c.d. of $a, b, c$ is 1 .

In real quadratic fields, there is a natural correspondence between classes of modules and $\mathrm{SL}_{2}(Z)$-equivalent classes of primitive quadratic forms. Let $M$ be a full module (module of rank 2 ) in a real quadratic field. The zeta function

Received by the editors December 16, 1987 and, in revised form, January 26, 1988.

1980 Mathematics Subject Classification (1985 Revision). Primary 11R42, 11M41; Secondary $11 \mathrm{E} 32$.

This work was supported by the Institute of Mathematics, Academia Sinica, and the National Science Foundation of Taiwan, Republic of China. 
of $M$ is defined by

$$
\zeta(M, s)=N(M)^{s} \sum_{\xi \in M / E} \frac{1}{N(\xi)^{s}}, \quad \operatorname{Re} s>1,
$$

where $E$ is the group of totally positive units $\varepsilon$ satisfying $\varepsilon M=M$, and $N$ is the norm on the real quadratic field. For any totally positive number $\lambda$, we have $\zeta(\lambda M, s)=\zeta(M, s)$. Hence $\zeta(M, s)$ can be considered as a zeta function associated with the module class $A$ to which $M$ belongs. Consequently, we write $\zeta(A, s)$ instead of $\zeta(M, s)$.

For a reduced quadratic form $Q(x, y)=a x^{2}+b x y+c y^{2}$, we define

$$
\begin{aligned}
Z_{Q}(s) & =\sum_{p=1}^{\infty} \sum_{q=1}^{\infty} \frac{1}{\left(a p^{2}+b p q+c q^{2}\right)^{s}}+\frac{1}{2} \sum_{p=1}^{\infty} \frac{1}{\left(a p^{2}\right)^{s}}+\frac{1}{2} \sum_{q=1}^{\infty} \frac{1}{\left(c q^{2}\right)^{s}} \\
& =Z_{Q}^{*}(s)+\frac{1}{2} \sum_{p=1}^{\infty} \frac{1}{\left(a p^{2}\right)^{s}}+\frac{1}{2} \sum_{q=1}^{\infty} \frac{1}{\left(c q^{2}\right)^{s}}, \quad \operatorname{Re} s>1 .
\end{aligned}
$$

In [16], Zagier proved that $\zeta(A, s)$ can be decomposed into finite combinations of $Z_{Q}(s)$, i.e.

$$
\zeta(A, s)=\sum_{Q} Z_{Q}(s)
$$

where the summation is over the reduced forms in the classes of forms associated to the module class of $M$. Also Zagier gave the values of $Z_{Q}(s)$ at nonpositive integers.

In this paper, we shall start with the zeta function

$$
\tilde{\zeta}_{2}(s)=\sum_{s_{1}=1}^{\infty} \sum_{s_{2}=1}^{\infty} \sum_{s_{12}=0}^{\infty} \frac{1}{\left[s_{1} s_{2}+\left(s_{1}+s_{2}\right) s_{12}\right]^{s}}, \quad \operatorname{Re} s>3 / 2,
$$

associated with the principal Delaunay-Voronoi cone as considered in [3]. Letting $s_{1}=p, s_{2}=q$, and $s_{12}=(a p+c q) /(b-a-c)$, we get the zeta function $Z_{Q}^{*}(s)$ up to a constant multiple $(b-a-c)^{s}$. With the method introduced in $[3,6,9]$, we get an integral expression for $Z_{Q}^{*}(s) \Gamma(s)(s-1 / 2) \pi^{1 / 2}$ when $\operatorname{Re} s \geq 3 / 2$, and the values of $Z_{Q}^{*}(s)$ at nonpositive integers and negative halfintegers can be written as a finite sum of integrals which are functions in $s$ and have analytic continuations in the whole complex plane.

Theorem 1. Let $m$ be a nonnegative integer. Then

$$
\begin{aligned}
& Z_{Q}^{*}(-m)=-\frac{(2 m+1) !}{2^{2 m}}(b-a-c)^{m} \frac{1}{2 \pi} N_{1}(-m), \\
& Z_{Q}^{*}\left(-m+\frac{1}{2}\right)=-\frac{B_{2 m}}{2^{2 m}}(b-a-c)^{m-(1 / 2)} \frac{1}{2 \pi} N_{2}\left(-m+\frac{1}{2}\right), \quad m \geq 1,
\end{aligned}
$$


where

$$
\begin{aligned}
& N_{1}(s)=\int_{0}^{1}\left(1-r^{2}\right)^{s-3 / 2} r d r \\
& \quad \cdot \int_{0}^{2 \pi} \sum_{p=0}^{m+1} \frac{B_{2 p} B_{2 m+2-2 p} R(r, \theta)^{2 p-1} T(r, \theta)^{2 m+1-2 p}}{2 p !(2 m+2-2 p) !}\left(+\frac{1}{4} \text { if } m=0\right) d \theta, \\
& N_{2}(s)=\int_{0}^{1}\left(1-r^{2}\right)^{s-3 / 2} r d r \int_{0}^{2 \pi}\left[R(r, \theta)^{2 m-1}+T(r, \theta)^{2 m-1}\right] d \theta,
\end{aligned}
$$

for $\operatorname{Re} s>1$, with

$$
\begin{aligned}
& R(r, \theta)=(1+r \sin \theta)+\frac{2 a}{b-a-c}(1-r \cos \theta), \\
& T(r, \theta)=(1-r \sin \theta)+\frac{2 c}{b-a-c}(1-r \cos \theta) .
\end{aligned}
$$

Here $B_{m}(m=0,1, \ldots)$ are Bernoulli numbers defined by

$$
\frac{t}{e^{t}-1}=\sum_{m=0}^{\infty} \frac{B_{m} t^{m}}{m !}, \quad|t|<2 \pi .
$$

Note that $N_{1}(s)$ has an analytic continuation which is holomorphic except for possible simple poles at $s=\frac{1}{2},-\frac{1}{2}, \ldots,-m+\frac{1}{2}$. On the other hand, $\mathrm{N}_{2}(s)$ has an analytic continuation which is holomorphic except for possible poles at $s=\frac{1}{2},-\frac{1}{2}, \ldots,-m+\frac{3}{2}$ if $-m+\frac{3}{2}<\operatorname{Re} s \leq-m+\frac{1}{2}$. Thus $N_{1}(-m)$ and $N_{2}\left(-m+\frac{1}{2}\right)$ can be obtained by the analytic continuation of $N_{1}(s)$ and $N_{2}(s)$. In particular, we have

Theorem 2. For positive integers $m$, we have

$$
\begin{aligned}
\frac{1}{2 \pi} N_{2}\left(-m+\frac{1}{2}\right)= & \sum_{l=0}^{m-1}\left(\begin{array}{c}
2 m-1 \\
2 l
\end{array}\right) \frac{[1 \cdot 3 \cdots(2 l-1)]}{[m(m-1) \cdots(m-l)](-2)^{l+1}} \\
& \cdot\left\{\left(1+\delta_{1}\right)^{2 m-1-2 l}\left(1+\delta_{1}^{2}\right)^{l}+\left(1+\delta_{2}\right)^{2 m-1-2 l}\left(1+\delta_{2}^{2}\right)^{l}\right\}
\end{aligned}
$$

with $\delta_{1}=2 a /(b-a-c)$ and $\delta_{2}=2 c /(b-a-c)$.

Consequently, by an elementary computation, we have the following:

$$
\begin{aligned}
Z_{Q}\left(-\frac{1}{2}\right)= & \frac{1}{24} \frac{b}{\sqrt{b-a-c}}-\frac{1}{24}(\sqrt{a}+\sqrt{c}) \\
Z_{Q}(-1)= & \frac{1}{24}\left(\frac{b}{a}+\frac{b}{c}\right)+\frac{1}{4} \\
Z_{Q}\left(-\frac{3}{2}\right)= & \frac{1}{1620} \cdot \frac{P(a, b, c)}{(b-a-c)^{3 / 2}}+\frac{1}{240}\left(a^{3 / 2}+c^{3 / 2}\right) \quad \text { with } \\
P(a, b, c)= & 6 a^{3}-b^{2}+6 c^{3}-3 a^{2} b-3 b c^{2} \\
& -6 a b^{2}-6 b^{2} c-6 a^{2} c-6 a c^{2}+30 a b c
\end{aligned}
$$




$$
Z_{Q}(-2)=\frac{1}{1440}\left(\frac{b^{3}-6 a b c}{a^{2}}+\frac{b^{3}-6 a b c}{c^{2}}\right)+\frac{b}{144}
$$

In particular, we prove the following result:

Theorem. Let $K$ be a real quadratic field of discriminant $D$ and denote by $G_{K}$ the (finite) set of positive divisors of integers of the form $\left(D-n^{2}\right) / 4 \quad(|n|<\sqrt{D}$, $n \equiv D \quad(\bmod 2))$. Then the value of the Dedekind zeta function of $K$, or of the zeta function of any ideal class of $K$, at a negative half-integral argument $s=1 / 2-m$ is a rational linear combination of the numbers $g^{1 / 2-m}\left(g \in G_{K}\right)$, the denominators of the coefficients being bounded by an integer depending only on $m$ ( 24 for $m=1,1620$ for $m=2, \ldots)$.

The above theorem is an easy consequence of Theorems 1 and 2 since the numbers $a, c$, and $b-a-c$ for any reduced form $a x^{2}+b x y+c y^{2}$ belong to $G_{K}$.

\section{The INTEgRAL EXPRESSION OF $Z_{Q}^{*}$ AND THE PROOF OF TheOREM 1}

Fix a reduced quadratic form $Q(x, y)=a x^{2}+b x y+c y^{2}$ and let

$$
Z_{Q}^{*}(s)=\sum_{p=1}^{\infty} \sum_{q=1}^{\infty} \frac{1}{\left(a p^{2}+b p q+c q^{2}\right)^{s}}, \quad \operatorname{Re} s>1
$$

In this section we shall obtain an integral expression for $Z_{Q}^{*}(s) \Gamma(s) \Gamma(s-1 / 2) \pi^{1 / 2}$ and the analytic continuation of this zeta function.

Lemma 1. Let $Y$ be the variable of a $2 \times 2$ real symmetric matrix and $G$ be a fixed $2 \times 2$ positive definite symmetric matrix. Then we have, for $\operatorname{Re} s \geq 3 / 2$,

$$
\int_{Y>0}(\operatorname{det} Y)^{s-3 / 2} e^{-\operatorname{tr}(Y G)} d Y=(\operatorname{det} G)^{-s} \pi^{1 / 2} \Gamma(s) \Gamma\left(s-\frac{1}{2}\right) .
$$

Here $\operatorname{tr} X=$ trace of $X$ for any matrix $X$.

Proof. See p. 225 of [1].

Proposition 1. For $\operatorname{Re} s \geq 3 / 2$, we have

$$
\Gamma(s) \Gamma\left(s-\frac{1}{2}\right) \pi^{1 / 2}(b-a-c)^{s} Z_{Q}^{*}(s)=\int_{Y>0}(\operatorname{det} Y)^{s-3 / 2} \frac{d Y}{\left(e^{A(Y)}-1\right)\left(e^{B(Y)}-1\right)},
$$

where

$$
Y=\left[\begin{array}{ll}
y_{1} & y_{12} \\
y_{12} & y_{2}
\end{array}\right] \text { and }\left\{\begin{array}{l}
A(Y)=y_{1}+\frac{a}{b-a-c}\left(y_{1}+y_{2}-2 y_{12}\right) \\
B(Y)=y_{2}+\frac{c}{b-a-c}\left(y_{1}+y_{2}-2 y_{12}\right)
\end{array}\right.
$$

Proof. Apply Lemma 1 with

$$
G=\left[\begin{array}{ll}
p & 0 \\
0 & q
\end{array}\right]+\frac{a p+b q}{b-a-c}\left[\begin{array}{cc}
1 & -1 \\
-1 & 1
\end{array}\right] \text {, }
$$


we get, for $\operatorname{Re} s \geq 3 / 2$,

$$
\begin{aligned}
\Gamma(s) \Gamma & \left(s-\frac{1}{2}\right) \pi^{1 / 2} Z_{Q}^{*}(s)(b-a-c)^{s}=\sum_{q=1}^{\infty} \sum_{p=1}^{\infty} \int_{Y>0}(\operatorname{det} Y)^{s-3 / 2} e^{-A(Y) p-B(Y) q} d Y \\
& =\int_{Y>0}(\operatorname{det} Y)^{s-3 / 2} \sum_{q=1}^{\infty} \sum_{p=1}^{\infty} e^{-A(Y) p-B(Y) q} d Y \\
& =\int_{Y>0}(\operatorname{det} Y)^{s-3 / 2} \frac{d Y}{\left(e^{A(Y)}-1\right)\left(e^{B(Y)}-1\right)} .
\end{aligned}
$$

Remark. Here the exchange of summation and integration is possible since the double series $\sum_{q=1}^{\infty} \sum_{p=1}^{\infty} e^{-A(Y) p-B(Y) q}$ is absolutely convergent and its partial sum is dominated by

$$
\frac{1}{\left(e^{A(Y)}-1\right)\left(e^{B(Y)}-1\right)}
$$

Proposition 2. $Z_{Q}^{*}(s)$ has an analytic continuation to the whole complex plane except a simple pole at $s=1 / 2$. Furthermore, we have

$$
Z_{Q}^{*}(s)=2 \Gamma(1-s) \frac{e^{-\pi i s}}{e^{2 \pi i s}+1} \cdot \frac{1}{2 \pi i} \int_{L(\varepsilon)} u^{2 s-3} I(s, u) d u
$$

where

$$
I(s, u)=\frac{1}{\Gamma(s-1 / 2) \pi^{1 / 2}} \int_{0}^{1}\left(1-r^{2}\right)^{s-3 / 2} r d r \int_{0}^{2 \pi} \frac{u^{2} d \theta}{\left(e^{R(r, \theta) u}-1\right)\left(e^{T(r, \theta) u}-1\right)},
$$

$L(\varepsilon)$ is the contour in the complex plane consisting of the interval $[\varepsilon,+\infty)$ twice, in both directions (in and out) and the circle $|z|=\varepsilon$ in counterclockwise direction, and

$$
\left\{\begin{array}{l}
R(r, \theta)=(1+r \sin \theta)+\frac{2 a}{b-a-c}(1-r \cos \theta), \\
T(r, \theta)=(1-r \sin \theta)+\frac{2 c}{b-a-c}(1-r \cos \theta) .
\end{array}\right.
$$

Proof. The first assertion was proved in [16]. Here we prove the integral expression from Proposition 1. By changing variables: $u=\left(y_{1}+y_{2}\right) / 2, v=$ $\left(y_{1}-y_{2}\right) / 2, w=y_{12}$, the integral expression for $\Gamma(s) \Gamma(s-1 / 2) Z_{Q}^{*}(s)(b-a-c)^{s}$ is transformed into

$$
2 \int_{u^{2}-v^{2}-w^{2}>0, u>0} \frac{\left(u^{2}-v^{2}-w^{2}\right)^{s-3 / 2} d u d v d w}{\left(e^{\left[u+v+\delta_{1}(u-w)\right]}-1\right)\left(e^{\left[u-v+\delta_{2}(u-w)\right]}-1\right)},
$$

where $\delta_{1}=2 a /(b-a-c)$ and $\delta_{2}=2 c /(b-a-c)$.

Let $v=u x, v=u y$ and then let $x=r \cos \theta, y=r \sin \theta$. It follows that

$$
\begin{aligned}
& \Gamma(s) \Gamma(s-1 / 2) \pi^{1 / 2 s} Z_{Q}^{*}(s)(b-a-c)^{s} \\
& \quad=2 \int_{0}^{\infty} u^{2 s-3} d u \int_{0}^{1}\left(1-r^{2}\right)^{s-3 / 2} r d r \int_{0}^{2 \pi} \frac{u^{2} d \theta}{\left(e^{R(r, \theta) u}-1\right)\left(e^{T(r, \theta) u}-1\right)} .
\end{aligned}
$$


As shown in [6], $I(s, u)$ has an analytic continuation which is a meromorphic function in $s$. The integration with respect to $u$ can be changed into a contour integral. Thus we have

$$
\Gamma(s) Z_{Q}^{*}(s)(b-a-c)^{s}=2\left(e^{4 \pi i s}-1\right)^{-1} \int_{L(\varepsilon)} u^{2 s-3} I(s, u) d u .
$$

In light of the functional equation for the gamma function

$$
\Gamma(s) \Gamma(1-s)=\frac{2 \pi i e^{\pi i s}}{e^{2 \pi i s}-1},
$$

we then have

$$
Z_{Q}^{*}(s)(b-a-c)^{s}=2 \Gamma(1-s) \frac{e^{-\pi i s}}{e^{2 \pi i s}+1} \cdot \frac{1}{2 \pi i} \int_{L(\varepsilon)} u^{2 s-3} I(s, u) d u .
$$

The contour integral is convergent for all $s$. Thus it defines the analytic continuation of $Z_{Q}^{*}(s)$.

Proof of Theorem 1. When $s=-m$ or $s=-m+\frac{1}{2} \quad(m>0)$, then $2 s-3$ is an integer. On the other hand, $I(s, u)$ is a holomorphic function in $u$. Consequently, the integrations along $[\varepsilon, \infty)$ twice in opposite directions will cancel and the evaluation of the contour integral is reduced to the calculation of residues of $u^{2 s-3} I(s, u)$ at $u=0$ and $s=-m$ or $-m+\frac{1}{2}$.

Note that

$$
\begin{array}{ll}
\frac{u}{e^{R(r, \theta) u}-1}=\frac{1}{R(r, \theta)}-\frac{1}{2}+\sum_{m=1}^{\infty} \frac{B_{2 m} u^{2 m} R(r, \theta)^{2 m-1}}{(2 m) !}, & |R(r, \theta) u|<2 \pi, \\
\frac{u}{e^{T(r, \theta) u}-1}=\frac{1}{T(r, \theta)}-\frac{1}{2}+\sum_{m=1}^{\infty} \frac{B_{2 m} u^{2 m} T(r, \theta)^{2 m-1}}{(2 m) !}, & |T(r, \theta) u|<2 \pi .
\end{array}
$$

By considering the coefficients of $u^{2 m+2}(s=-m)$ and $u^{2 m+1} \quad\left(s=-m+\frac{1}{2}\right)$ in the power expansion of

$$
\frac{u^{2}}{\left(e^{R(r, \theta) u}-1\right)\left(e^{T(r, \theta) u}-1\right)}
$$

at $u=0$, we get our assertion for $Z_{Q}^{*}(-m)$ and $Z_{Q}^{*}\left(-m+\frac{1}{2}\right)$ as listed in Theorem 1 of the Introduction.

Remark. Here we use the following identities, which can be verified in an elementary way:

$$
\begin{gathered}
\lim _{s \rightarrow-m} 2 \Gamma(1-s) \frac{e^{-\pi i s}}{e^{2 \pi i s}+1} \cdot \frac{1}{\Gamma\left(s-\frac{1}{2}\right) \pi^{1 / 2}}=-\frac{(2 m+1) !}{2^{2 m}} \cdot \frac{1}{2 \pi}, \\
\lim _{s \rightarrow-m+1 / 2} 2 \Gamma(1-s) \frac{e^{-\pi i s}}{e^{2 \pi i s}+1} \cdot \frac{1}{\Gamma\left(s-\frac{1}{2}\right) \pi^{1 / 2}}=-\frac{(2 m) !}{2^{2 m} \pi}
\end{gathered}
$$




\section{THE PROOF OF THEOREM 2}

The evaluation of $N_{1}(-m)$ and $N_{2}\left(-m+\frac{1}{2}\right)$ can be done by the same arguments as in [3]. However, as the values of $Z_{Q}(s)$ at nonpositive integers were given in [16], it is unnecessary to compute $N_{1}(-m)$ (though it is possible). Here we only compute the value of $N_{2}\left(-m+\frac{1}{2}\right)$.

Proof of Theorem 2. Note that

$$
1+r \sin \theta+\delta_{1}(1-r \cos \theta)=1+\delta_{1}+\sqrt{1+\delta_{1}^{2}} r \sin (\theta-\phi)
$$

with $\phi=\tan ^{-1}\left(1 / \delta_{1}\right)$. Hence

$$
\begin{aligned}
\int_{0}^{2 \pi} & R(r, \theta)^{2 m-1} d \theta=\int_{0}^{2 \pi}\left[1+\delta_{1}+\sqrt{1+\delta_{1}^{2}} r \sin (\theta-\phi)\right]^{2 m-1} d \theta \\
& =\int_{0}^{2 \pi}\left[1+\delta_{1}+\sqrt{1+\delta_{1}^{2}} r \sin \theta\right]^{2 m-1} d \theta \\
& =\sum_{l=0}^{m-1}\left(\begin{array}{c}
2 m-1 \\
2 l
\end{array}\right)\left(1+\delta_{1}\right)^{2 m-1-2 l}\left(1+\delta_{1}^{2}\right)^{l} \int_{0}^{2 \pi} r^{2 l} \sin ^{2 l} \theta d \theta .
\end{aligned}
$$

For sufficiently large $s$, we have

$$
\int_{0}^{1} \int_{0}^{2 \pi}\left(1-r^{2}\right)^{s-3 / 2} r^{2 l+1} \sin ^{2 l} \theta d \theta d r=\frac{[1 \cdot 3 \cdots(2 l-1)] 2 \pi}{(2 s-1)(2 s+1) \cdots(2 s-1+2 l)} .
$$

Thus the contribution from $R(r, \theta)^{2 m-1}$ to $(1 / 2 \pi) N_{2}(-m)$ is given by

$$
\begin{aligned}
& \sum_{l=0}^{m-1}\left(\begin{array}{c}
2 m-1 \\
2 l
\end{array}\right)\left(1+\delta_{1}\right)^{2 m-1-2 l}\left(1+\delta_{1}^{2}\right) \frac{[1 \cdot 3 \cdots(2 l-1)]}{[(-2 m)(-2 m+2) \cdots(-2 m+2 l)]} \\
& =\sum_{l=0}^{m-1}\left(\begin{array}{c}
2 m-1 \\
2 l
\end{array}\right)\left(1+\delta_{1}\right)^{2 m-1-2 l}\left(1+\delta_{1}^{2}\right)^{l} \frac{[1 \cdot 3 \cdots(2 l-1)]}{m(m-1) \cdots(m-l)](-2)^{l+1}} .
\end{aligned}
$$

In the same way, we get the contribution from $T(r, \theta)^{2 m-1}$ to

$$
(1 / 2 \pi) N_{2}(-m) \text {. }
$$

Corollary. Let $m$ be a positive integer. Then

$$
\begin{aligned}
Z_{Q}\left(-m+\frac{1}{2}\right)= & -\frac{B_{2 m}}{2^{2 m}} \sum_{l=0}^{m-1}\left(\begin{array}{c}
2 m-1 \\
2 l
\end{array}\right) \frac{[1 \cdot 3 \cdots(2 l-1)]}{[m(m-1) \cdots(m-l)](-2)^{l+1}} \\
& \cdot\left\{\left(1+\delta_{1}\right)^{2 m-1-2 l}\left(1+\delta_{1}^{2}\right)^{l}+\left(1+\delta_{2}\right)^{2 m-1-2 l}\left(1+\delta_{2}^{2}\right)^{l}\right\} \\
& \cdot(b-a-c)^{m-1 / 2}-\frac{1}{2}\left(a^{m-1 / 2}+c^{m-1 / 2}\right) \frac{B_{2 m}}{2 m}
\end{aligned}
$$

where $\delta_{1}=2 a /(b-a-c), \delta_{2}=2 c /(b-a-c)$. 


\section{REFERENCES}

1. W. L. Baily, Jr., Introductory lectures on automorphic forms, Princeton Univ. Press, 1973.

2. Minking Eie, A zeta-function associated with zero ternary forms, Proc. Amer. Math. Soc. 94 (1985), 387-392.

3. Minking Eie and Chong-hsio Fang, On the residues and values of a zeta function at negative integers and negative half-integers, manuscript, 1987.

4. I. M. Gelfand and G. E. Shilov, Generalized functions, vol. 1, 1964.

5. David Kramer, On the values of integers of the Dedekind zeta function of a real quadratic field, Trans. Amer. Math. Soc. 299 (1987), 59-79.

6. A. Kurihara, On the values of nonpositive integers of Siegel's zeta functions of $Q$-anisotropic quadratic forms with signature $(1, n-1)$, J. Fac. Sci. Univ. Tokyo, Sect. 1A Math. 28 (1981), 567-584.

7. Y. Namikawa, Toroidal compactification of Siegel spaces, Lecture Notes in Math., vol. 812, Springer-Verlag, Berlin and New York,

8. __, A new compactification of the Siegel space and degeneration of abelian varieties. I, Math. Ann. 221 (1976), 97-141.

9. I. Satake, Special values of zeta functions associated with self-dual homogeneous cones, manuscript, 1981.

10. M. Sato, and T. Shintani, On zeta functions associated with prehomogeneous vector spaces, Ann. of Math. (2) 100 (1974), 131-170.

11. T. Shintani, Zeta-functions associated with the vector of quadratic forms, J. Fac. Sci. Univ. Tokyo Sect. 1A Math. 22 (1975), 25-65.

12. __ On evaluation of zeta functions of totally real algebraic number fields at nonpositive integers, J. Fac. Sci. Univ Tokyo 23 (1976), 393-417.

13. C. L. Siegel, Über die analytische Theorie der quadratischen Formen, Ann. of Math. (2) 36 (1935), 527-606.

14. _ـ Über die Zetafunktionen indefiniter quadratischer Formen, Math. Z. 43 (1938), 682-708.

15. D. Zagier, A Kronecker limit formula for real quadratic fields, Math. Ann. 213 (19), 153-184.

16. __ Valeurs des fonctions zeta des corps quadratiques reèls aux entiers negatifs, J. Arithmétiques de Caen, Astérisque 41-42 (1977), 135-151.

Institute of Mathematics, Academia Sinica, Nankang, Taipei 11529, Taiwan, Republic OF CHINA 